\title{
A Galerkin Method for a Gaseous Ignition Model
}

\section{Salman* and J. Kim*}

${ }^{*}$ Department of Mathematics, Statistics and Physics, University of Qatar, Doha, Qatar, Email: msalman@qu.edu.qa.

${ }^{* \star D e p a r t m e n t}$ of Mathematics, Tuskegee University, Tuskegee, Alabama, USA, Email: jtkim@tuskegee.edu.

\begin{abstract}
We consider a Galerkin procedure to solve a parabolic integrodifferential equation that arises in a gas combustion model. This model has been proposed by Kassoy and Poland, and subsequently analyzed by Bebernes, Eberly and Bressan. The problem is formulated in the variational form. In order to estimate the error, some intermediate projection has been employed. Under certain conditions on the given data, the $L^{2}$ error estimate has been obtained. A fully descretized version by using an extrapolated Crank-Nicolson method has been applied and the order of convergence derived.
\end{abstract}

KEYWORDS: Crank-Nicolson, Error estimate, Galerkin method, Gaseous ignition model.

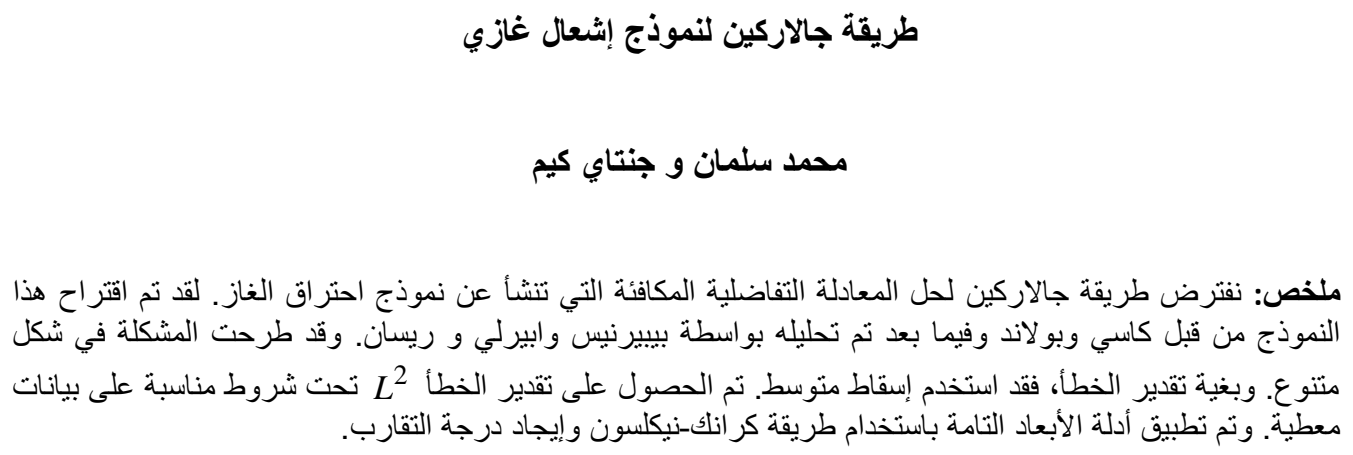

\section{Introduction}

Tassoy and Poland (1983) developed an ignition model for a reactive gas in a bounded container to describe Ithe induction period. During this period, the spacially uniform pressure increases and causes heating effects in the system. The pressure of the gas can be expressed in terms of a space integral term in the induction model that governs the temperature perturbation $u(x, t)$. This model is described by the set of equations (Bebernes and Bressan, 1988)

$$
u_{t}-\Delta u=\delta e^{u}+\frac{\gamma-1}{\gamma} \frac{1}{|\Omega|} \int_{\Omega} u_{t}(x, t) d x, \quad(x, t) \in \Omega \times(0, \infty),
$$




\section{A GALERKIN METHOD FOR A GASEOUS IGNITION MODEL}

$$
\begin{gathered}
u(x, 0)=g(x), \quad x \in \Omega, \\
u(x, t)=0, \quad(x, t) \in \partial \Omega \times(0, \infty),
\end{gathered}
$$

where $\Omega$ is a bounded domain in $\mathbb{R}^{n}$ with a smooth boundary $\partial \Omega$, and volume $|\Omega|, \gamma>1$.

The model has been subsequently studied by Bebernes and Bressan (1982), Bebernes and Bressan (1988), and Bebernes et al. (1989). Bebernes and Bressan (1982) analyzed this model and proved that for any positive value of the Frak-Kamenetski parameter $\delta$ and any value of the gas constant $\gamma \geq 1$, equations (1) have a unique classical solution $u(x, t)$ on $\Omega \times[0, T)$, where $\Omega$ is a bounded domain and $T$ can be infinite. When $T$ is finite, the solution blows up as $t \rightarrow T$. For a critical value $\delta_{\text {crit }}$ (see (Kassoy and Poland, 1983)), and $\delta>\delta_{\text {crit }}$, the solution blows up in a finite time.

Bebernes and Eberly (1989) used the semigroup analysis to show the existence and uniqueness of a nonextended solution. Additional comparison results have been provided in the case of a spherically symmetric domain. Blowup occurs at a time $\sigma<T$ where $T$ is the blowup time of the solid fuel ignition model. The location of the blowup has been also discussed. Depending on the nonlinearity of $f$, blowup can take place everywhere or at a single point (Bebernes and Eberly, 1989).

In this paper, we study a finite element approximation to the solution of the gas combustion model that is described by the partial differential equation (Bebernes and Eberly, 1989)

$$
\begin{aligned}
u_{t}-\Delta u & =f(u)+\frac{\gamma-1}{\gamma} \frac{1}{|\Omega|} \int_{\Omega} u_{t}(x, t) d x, \quad(x, t) \in \Omega \times(0, \infty), \\
u(x, 0) & =g(x), \quad x \in \Omega, \\
u(x, t) & =0, \quad(x, t) \in \partial \Omega \times(0, \infty) .
\end{aligned}
$$

We assume $f$ is a Lipschitz function such that $f(u)>0, f^{\prime}(u) \geq 0$, and $f^{\prime \prime}(u) \geq 0$. In this work we develop estimates for error when a Galerkin method is applied. The error is optimal in the sense of the $L^{2}$ norm. This work is motivated by that of Cannon and Lin (1990a, 1990b). An extensive study of the finite element method for parabolic equations can be found in a book by Thomée (2006).

\section{Formulation of the variational problem and Galerkin approximation}

Let $S_{h}$ be a finite dimensional subspace of the Sobolev space $H_{0}^{1}(\Omega)$ such that

$$
\inf _{w \in S_{h}}(\|w-v\|+h\|\nabla(w-v)\|) \leq C h^{s}\|v\|_{s}, \quad v \in H^{s}(\Omega) \cap H_{0}^{1}(\Omega),
$$

where $s \geq 1,\|\cdot\|$ is the $L^{2}$ norm, and $\|\cdot\|_{s}$ is the Sobolev norm defined on $H^{s}(\Omega)$.

Problem (4) is equivalent to finding a $u \in H^{s}(\Omega) \cap H_{0}^{1}(\Omega)$ such that

$$
\left(u_{t}, v\right)+(\nabla u, \nabla v)=(f(u), v)+\frac{\gamma-1}{\gamma} \frac{1}{|\Omega|} \int_{\Omega} u_{t} d x \int_{\Omega} v d x, \quad \text { for all } v \in H_{0}^{1}(\Omega),
$$

where $(\cdot, \cdot)$ is the inner product on $L^{2}(\Omega)$ defined as $(u, v)=\int_{\Omega} u v d x$.

The continuous Galerkin approximation $U:[0, T] \rightarrow S_{h}$ is defined as a solution to

$$
\begin{aligned}
\left(U_{t}, \chi\right)+(\nabla U, \nabla \chi) & =(f(U), \chi)+\frac{\gamma-1}{\gamma} \frac{1}{|\Omega|} \int_{\Omega} U_{t} d x \int_{\Omega} \chi d x, \quad \chi \in S_{h} \\
U(x, 0) & =G(x),
\end{aligned}
$$

where $G(x)$ is the $L^{2}$ projection of $g(x)$ into $S_{h}$, i.e., 


\section{SALMAN and J. KIM}

$$
(G-g, \chi)=0 \text { for } \chi \in S_{h} .
$$

Given a basis $\left\{\phi_{i}\right\}_{i=1}^{M}$ for $S_{h}, U$ can be written as

$$
U(x, t)=\sum_{i=1}^{M} \alpha_{i}(t) \phi_{i}(x) .
$$

Then the variational equation can be written as the nonlinear initial value problem

$$
B \mathbf{a}^{\prime}(t)+A \mathbf{a}(t)=F(\mathbf{a}(t)), \quad C \mathbf{a}(0)=\mathbf{g},
$$

where $A, B$, and $C$ are the matrices

$$
\begin{aligned}
& A=\left(\nabla \phi_{i}, \nabla \phi_{j}\right), \\
& B=\left(b_{i j}\right)=\left(\phi_{i}, \phi_{j}\right)-\frac{\gamma-1}{\gamma} \frac{1}{|\Omega|} \int_{\Omega} \phi_{i} d x \int_{\Omega} \phi_{i} d x, \\
& C=\left(\phi_{i}, \phi_{j}\right),
\end{aligned}
$$

for $i, j=1,2, \cdots, M$, and the vectors $\mathbf{a}, \mathbf{F}$, and $\mathbf{g}$ are defined by

$$
\begin{aligned}
& \mathbf{a}(t)=\left(\alpha_{i}(t)\right), \\
& \mathbf{F}(\mathbf{a})=\left(f\left(\sum_{i=1}^{M} \alpha_{i} \phi_{i}\right), \phi_{j}\right), \\
& \mathbf{g}=\left(g, \phi_{i}\right) .
\end{aligned}
$$

The matrix $B$ is positive definite, since

$$
\begin{aligned}
\mathbf{a}^{T} B \mathbf{a} & =\sum_{i=1}^{M} b_{i j} \alpha_{i} \alpha_{j} \\
& =\left\|\sum_{i=1}^{M} \alpha_{i} \phi_{i}\right\|^{2}-\frac{\gamma-1}{\gamma} \frac{1}{|\Omega|}\left(\int_{\Omega} \sum_{i=1}^{M} \alpha_{i} \phi_{i} d x\right)^{2} \\
& =\|U\|^{2}-\frac{\gamma-1}{\gamma} \frac{1}{|\Omega|}\left(\int_{\Omega} U d x\right)^{2} \\
& \geq \frac{1}{\gamma}\|U\|^{2}>0 \text { for } U \neq 0,
\end{aligned}
$$

where we used the Schwarz inequality $\left(\int_{\Omega} U d x\right)^{2} \leq \int_{\Omega} U^{2} d x \int_{\Omega} 1^{2} d x=|\Omega|\|U\|^{2}$.

With the assumption that $f(u)$ is uniformly Lipschitz, then it follows from the theory of ordinary differential equations that the initial-value problem (10) has a unique solution for $t>0$.

\section{Projection of the solution}

Let $W:[0, T] \rightarrow S_{h}$ such that

$$
(\nabla(u-W), \nabla \chi)=0 \text { for all } \quad \chi \in S_{h} .
$$

Then $W$ is the elliptic projection of $u \in H^{s}(\Omega) \cap H_{0}^{1}(\Omega)$ into $S_{h}$ that satisfies the following properties (Thomée, 2006)

$$
\begin{gathered}
\|u-W\| \leq C h^{s}\|u\|_{s}, \\
\|\nabla u-\nabla W\| \leq C h^{s-1}\|u\|_{s}, \\
\left\|u_{t}-W_{t}\right\| \leq C h^{s}\|u\|_{s} .
\end{gathered}
$$




\section{A GALERKIN METHOD FOR A GASEOUS IGNITION MODEL}

\section{Error estimates}

Let $u-U=u-W+W-U=\eta+\theta$, where $\eta=u-W$ and $\theta=W-U$. From (8), (9) and (11), we get

$$
\begin{aligned}
\left(\theta_{t}, \chi\right)+(\nabla \theta, \nabla \chi) & =\left(W_{t}-U_{t}, \chi\right)+(\nabla W-\nabla U, \nabla \chi) \\
& =\left(W_{t}, \chi\right)+(\nabla W, \nabla \chi)-\left(U_{t}, \chi\right)-(\nabla U, \nabla \chi) \\
& =\left(W_{t}, \chi\right)+(\nabla u, \nabla \chi)-(f(U), \chi)-\frac{\gamma-1}{\gamma} \frac{1}{|\Omega|} \int_{\Omega} U_{t} d x \int_{\Omega} \chi d x \\
& =\left(W_{t}-u_{t}, \chi\right)+(f(u)-f(U), \chi)+\frac{\gamma-1}{\gamma} \frac{1}{|\Omega|} \int_{\Omega}\left(u_{t}-U_{t}\right) d x \int_{\Omega} \chi d x,
\end{aligned}
$$

i.e.,

$\left(\theta_{t}, \chi\right)+(\nabla \theta, \nabla \chi)=-\left(\eta_{t}, \chi\right)+(f(u)-f(U), \chi)+\frac{\gamma-1}{\gamma} \frac{1}{|\Omega|} \int_{\Omega} \eta_{t} d x \int_{\Omega} \chi d x+\frac{\gamma-1}{\gamma} \frac{1}{|\Omega|} \int_{\Omega} \theta_{t} d x \int_{\Omega} \chi d x$

We choose $\chi=\theta$ and rewrite the equation

$\frac{1}{2} \frac{d}{d t}\|\theta\|^{2}-\frac{1}{2} \frac{\gamma-1}{\gamma} \frac{1}{|\Omega|} \frac{d}{d t}\left(\int_{\Omega} \theta d x\right)^{2}+\|\nabla \theta\|^{2}=-\left(\eta_{t}, \theta\right)+(f(u)-f(U), \theta)+\frac{\gamma-1}{\gamma} \frac{1}{|\Omega|} \int_{\Omega} \eta_{t} d x \int_{\Omega} \theta d x$.

Assuming that $f$ is uniformly Lipschitz with

$$
\left|f\left(u_{1}\right)-f\left(u_{2}\right)\right| \leq L\left|u_{1}-u_{2}\right| .
$$

Then, using Schwarz and Young's inequalities implies

$$
\frac{1}{2} \frac{d}{d t}\|\theta\|^{2}-\frac{1}{2} \frac{\gamma-1}{\gamma} \frac{1}{|\Omega|} \frac{d}{d t}\left(\int_{\Omega} \theta d x\right)^{2}+\|\nabla \theta\|^{2} \leq \frac{C}{\varepsilon}\left\|\eta_{t}\right\|^{2}+C \varepsilon\|\theta\|^{2}+L\|\theta\|^{2}+L\|\eta\|\|\theta\| .
$$

With the use of Poincaré-Friedrichs' inequality (Gilbarg and Trudinger, 1983)

$$
\|u\| \leq\left(\frac{|\Omega|}{\omega_{n}}\right)^{1 / n}\|\nabla u\|
$$

we obtain

$$
\frac{1}{2} \frac{d}{d t}\|\theta\|^{2}-\frac{1}{2} \frac{\gamma-1}{\gamma} \frac{1}{|\Omega|} \frac{d}{d t}\left(\int_{\Omega} \theta d x\right)^{2}+\left(\frac{|\Omega|}{\omega_{n}}\right)^{2 / n}\|\theta\|^{2} \leq \frac{C}{\varepsilon}\left\|\eta_{t}\right\|^{2}+\frac{C}{\varepsilon}\|\eta\|^{2}+(L+C \varepsilon)\|\theta\|^{2} .
$$

If the Lipschitz constant of $f$ is small enough such that

$$
L<\left(\frac{|\Omega|}{\omega_{n}}\right)^{2 / n},
$$

then we can also choose $\varepsilon$ small enough so that $\left(\frac{|\Omega|}{\omega_{n}}\right)^{2 / n} \geq L+C \varepsilon$. Thus, we have

$$
\frac{1}{2} \frac{d}{d t}\|\theta\|^{2}-\frac{1}{2} \frac{\gamma-1}{\gamma} \frac{1}{|\Omega|} \frac{d}{d t}\left(\int_{\Omega} \theta d x\right)^{2}+C\|\theta\|^{2} \leq C\|\eta\|^{2}+C\left\|\eta_{t}\right\|^{2}
$$

Integrating both sides from 0 to $t$ after dropping $C\|\theta\|^{2}$ to get

$$
\|\theta\|^{2}-\frac{\gamma-1}{\gamma} \frac{1}{|\Omega|}\left(\int_{\Omega} \theta d x\right)^{2} \leq\|\theta(\cdot, 0)\|^{2}+C \int_{0}^{t}\left(\|\eta\|^{2}+\left\|\eta_{t}\right\|^{2}\right) d \tau .
$$

Then, using schwarz inequality to obtain

$$
\frac{1}{\gamma|\Omega|}\|\theta\|^{2} \leq\|\theta(\cdot, 0)\|^{2}+C \int_{0}^{t}\left(\|\eta\|^{2}+\left\|\eta_{t}\right\|^{2}\right) d \tau .
$$




\section{SALMAN and J. KIM}

Here

$$
\begin{aligned}
\|\theta(\cdot, 0)\|^{2} & =\|W(\cdot, 0)-U(\cdot, 0)\| \\
& \leq\|W(\cdot, 0)-u(\cdot, 0)\|+\|u(\cdot, 0)-U(\cdot, 0)\| \\
& \leq C h^{s}\|g\|_{S}+\|g-G\| \leq C h^{s}\|g\|_{S},
\end{aligned}
$$

and $\|\eta\|^{2}+\left\|\eta_{t}\right\|^{2}$ can be replaced by their upper bound in (12) and (14). This implies

$$
\|u-U\| \leq C h^{s}\|g\|_{s}+C h^{s} \int_{0}^{t}\left(\|u\|_{S}+\left\|u_{t}\right\|_{s}\right) d \tau .
$$

This establishes the following theorem.

Theorem 1. Suppose that problem (4) possesses a solution $u$ in $H^{s}(\Omega) \cap H_{0}^{1}(\Omega)$, $u_{t}$ in $H^{s}(\Omega)$, and $f$ is uniformly Lipschitz that satisfies (17) and (20). Then, the continuous Galerkin solution $U$ of (9) satisfies (22).

Proof. The next step is to get an estimate for $\nabla(u-W)$. For that purpose we put $\chi=\theta_{t}$ in (15). This yields

$$
\left\|\theta_{t}\right\|^{2}+\frac{1}{2} \frac{d}{d t}\|\nabla \theta\|^{2}=-\left(\eta_{t}, \theta_{t}\right)+\left(f(u)-f(U), \theta_{t}\right)+\frac{\gamma-1}{\gamma} \frac{1}{|\Omega|} \int_{\Omega} \eta_{t} d x \int_{\Omega} \theta_{t} d x+\frac{\gamma-1}{\gamma} \frac{1}{|\Omega|}\left(\int_{\Omega} \theta_{t} d x\right)^{2} .
$$

This implies

$$
\left\|\theta_{t}\right\|^{2}+\frac{1}{2} \frac{d}{d t}\|\nabla \theta\|^{2} \leq \frac{C}{\varepsilon}\left\|\eta_{t}\right\|^{2}+C \varepsilon\left\|\theta_{t}\right\|^{2}+\frac{C}{\varepsilon}\|f(u)-f(U)\|^{2}+\frac{\gamma-1}{\gamma}\left\|\theta_{t}\right\|^{2} .
$$

Estimating the righthand side we get

$$
\frac{1}{\gamma}\left\|\theta_{t}\right\|^{2}+\frac{1}{2} \frac{d}{d t}\|\nabla \theta\|^{2} \leq \frac{C}{\varepsilon}\left\|\eta_{t}\right\|^{2}+C \varepsilon\left\|\theta_{t}\right\|^{2}+\frac{C L^{2}}{\varepsilon}\|u-U\|^{2} .
$$

Selecting $\varepsilon$ small so that $\frac{1}{\gamma}>C \varepsilon$, we can drop the $\left\|\theta_{t}\right\|$ terms to get

$$
\frac{d}{d t}\|\nabla \theta\|^{2} \leq C\left\|\eta_{t}\right\|^{2}+C\|u-U\|^{2}
$$

Upon integrating from 0 to $t$, we get

$$
\left.\|\nabla \theta\|^{2} \leq\|\nabla \theta(\cdot, 0)\|^{2}+C \int_{0}^{t}\left\|\eta_{t}\right\|^{2}+C \int_{0}^{t}\|u-U\|^{2}\right) d \tau,
$$

where

$$
\begin{aligned}
\|\nabla \theta(\cdot, 0)\| & \leq\|\nabla u(\cdot, 0)-\nabla U(\cdot, 0)\|+\|\nabla W(\cdot, 0)-\nabla u(\cdot, 0)\| \\
& \leq\|\nabla g-\nabla G\|+C h^{s-1}\|g\|_{s-1},
\end{aligned}
$$

and

$$
\int_{0}^{t}\|u-U\| d \tau \leq \int_{0}^{t}\left(C h^{s-1}\left\|g_{s-1}\right\|_{s-1}+\|g-G\|+C h^{s-1} \int_{0}^{\tau}\left(\|u(\cdot, \beta)\|_{s}+\left\|u_{t}(\cdot, \beta)\right\|_{s}\right) d \beta\right) d \tau .
$$

The double integration can be interchanged, a process to suppress one of the integrals, then the right hand side simplifies to

$$
\int_{0}^{t}\|u-U\| d \tau \leq C \int_{0}^{t}\left[\|g-G\|+h^{s-1}\left(\|g\|_{s-1}+\|u\|_{s-1}+\left\|u_{t}\right\|_{s-1}\right)\right] d \tau .
$$

In view of (28) and (30), estimate (27) may become

$$
\|\nabla \theta\|^{2} \leq\|\nabla g-\nabla G\|+C h^{s-1}\|g\|_{s-1}+C \int_{0}^{t}\left[\|g-G\|+h^{s-1}\left(\|g\|_{s-1}+\|u\|_{s-1}+\left\|u_{t}\right\|_{s-1}\right)\right] d \tau .
$$

This proves the theorem. 


\section{A GALERKIN METHOD FOR A GASEOUS IGNITION MODEL}

Theorem 2. Under all the assumptions mentioned in Theorem 1, we have

$$
\|\nabla u-\nabla U\| \leq C h^{s-1}\left\{\|g\|_{s-1}+\|u\|_{s-1}+\int_{0}^{t}\left(\|u\|_{s-1}+\left\|u_{t}\right\|_{s-1}\right) d \tau\right\} .
$$

Note that as $G$ being the $L^{2}$ projection of $g$ onto $S_{h}$, , it legitimizes the estimates

$$
\begin{gathered}
\|g-G\| \leq C h^{s}\|g\|_{S}, \\
\|\nabla g-\nabla G\| \leq C h^{s-1}\|g\|_{s-1} .
\end{gathered}
$$

\section{A priori estimate on extrapolated Crank-Nicolson-Galerkin method}

In order to get a fully discretized version of the Galerkin method, we introduce the time mesh $t_{m}=m k$ for $m=0,1, \cdots, M$, where $k$ is a uniform time step. For the rest of this section, we denote

$$
\bar{F}_{m}=\frac{1}{2}\left(F_{m}+F_{m+1}\right)
$$

as an averaged value of $F$ on the nodes $t_{m}$ and $t_{m+1}$.

In the Crank-Nicolson method, we replace the time derivative in (9) by $\partial U_{m}=\left(U_{m+1}-U_{m}\right) / k$ and $U$ by $\bar{U}_{m}=\left(U_{m}+U_{m+1}\right) / 2$. This defines $U_{m+1}$ as a solution to the nonlinear system

$$
\left(\partial U_{m}, \chi\right)+\left(\nabla \bar{U}_{m}, \nabla \chi\right)=\left(f\left(\bar{U}_{m}\right), \chi\right)+\frac{\gamma-1}{\gamma} \frac{1}{|\Omega|} \int_{\Omega} \partial U_{m} d x \int_{\Omega} \chi d x, \quad \chi \in S_{h} .
$$

The nonlinearity due to $f\left(\bar{U}_{m}\right)$ can be overcome by replacing the argument of $f\left(\bar{U}_{m}\right)$ by an extrapolated $U$ over the time steps $m$ and $m-1$, i.e.

We denote these extrapolated values by

$$
f\left(\bar{U}_{m}\right) \approx f\left(\frac{3}{2} U_{m}-\frac{1}{2} U_{m-1}\right) .
$$

$$
\hat{F}_{m}=\frac{3}{2} F_{m}-\frac{1}{2} F_{m-1} .
$$

This produces the new linearized equation in $U_{m+1}$ as

$$
\left(\partial U_{m}, \chi\right)+\left(\nabla \bar{U}_{m}, \nabla \chi\right)=\left(f\left(\hat{U}_{m}\right), \chi\right)+\frac{\gamma-1}{\gamma} \frac{1}{|\Omega|} \int_{\Omega} \partial U_{m} d x \int_{\Omega} \chi d x, \quad \chi \in S_{h} .
$$

Note that this extrapolation process will result in a second order accuracy

$$
\hat{u}_{m}=\frac{3}{2} u_{m}-\frac{1}{2} u_{m-1}=u_{m+1 / 2}+O\left(k^{2}\right),
$$

with $u_{m+1 / 2}=u\left(., t_{m+1 / 2}\right)$. We shall estimate the error $\left\|U_{m}-u\left(\cdot, t_{m}\right)\right\|$

$$
U_{m}-u\left(\cdot, t_{m}\right)=U_{m}-W\left(\cdot, t_{m}\right)+W\left(\cdot, t_{m}\right)-u\left(\cdot, t_{m}\right)=\theta_{m}+\eta_{m},
$$

where the estimate of $\eta_{m}$ is shown in (12). We now consider $\theta_{m}$ by writing

$$
\begin{aligned}
\left(\partial \theta_{m}, \chi\right)+\left(\nabla \bar{\theta}_{m}, \nabla \chi\right) & =\left(\partial U_{m}, \chi\right)+\left(\nabla \bar{U}_{m}, \nabla \chi\right)-\left(\partial W_{m}, \chi\right)+\left(\bar{W}_{m}, \nabla \chi\right) \\
& =\left(f\left(\hat{U}_{m}\right)-\bar{f}\left(u_{m}\right), \chi\right)+\frac{\gamma-1}{\gamma} \frac{1}{|\Omega|} \int_{\Omega}\left(\partial U_{m}-\frac{\partial \bar{u}_{m}}{\partial t}\right) d x \int_{\Omega} \chi d x+\left(\frac{\partial \bar{u}_{m}}{\partial t}-\partial W_{m}, \chi\right)
\end{aligned}
$$

where 


\section{SALMAN and J. KIM}

$$
\bar{f}\left(u_{m}\right)=\frac{1}{2}\left(f\left(u_{m}\right)+f\left(u_{m+1}\right)\right) .
$$

This implies

$$
\begin{aligned}
& \left(\partial \theta_{m}, \chi\right)-\frac{\gamma-1}{\gamma} \frac{1}{|\Omega|} \int_{\Omega} \partial \theta_{m} d x \int_{\Omega} \chi d x+\left(\nabla \bar{\theta}_{m}, \nabla \chi\right) \\
& \quad=\left(f\left(\hat{U}_{m}\right)-\bar{f}\left(u_{m}\right), \chi\right)+\frac{\gamma-1}{\gamma} \frac{1}{|\Omega|} \int_{\Omega}\left(\partial \eta_{m}+\partial u_{m}-\frac{\partial \bar{u}_{m}}{\partial t}\right) d x \int_{\Omega} \chi d x-\left(\partial \eta_{m}+\partial u_{m}-\frac{\partial \bar{u}_{m}}{\partial t}, \chi\right) .
\end{aligned}
$$

Setting $\chi=\bar{\theta}_{m}$, we can get

$$
\frac{1}{2} \partial\left\|\theta_{m}\right\|^{2}-\frac{\gamma-1}{2 \gamma} \frac{1}{|\Omega|} \partial\left(\int_{\Omega} \theta_{m} d x\right)^{2}+\left\|\nabla \bar{\theta}_{m}\right\|^{2} \leq C\left(\left\|f\left(\hat{U}_{m}\right)-\bar{f}\left(u_{m}\right)\right\|+\left\|\partial \eta_{m}\right\|+\left\|\partial u_{m}-\frac{\partial \bar{u}_{m}}{\partial t}\right\|\right)\left\|\nabla \bar{\theta}_{m}\right\|,
$$

where we have used the Poincaré-Friedrichs' inequality $\left\|\bar{\theta}_{m}\right\| \leq\left\|\nabla \bar{\theta}_{m}\right\|$. This implies

$$
\frac{1}{2} \partial\left\|\theta_{m}\right\|^{2}-\frac{\gamma-1}{2 \gamma} \frac{1}{|\Omega|} \partial\left(\int_{\Omega} \partial \theta_{m} d x\right)^{2} \leq C\left(\left\|f\left(\hat{U}_{m}\right)-\bar{f}\left(u_{m}\right)\right\|^{2}+\left\|\partial \eta_{m}\right\|^{2}+\left\|\partial u_{m}-\frac{\partial \bar{u}_{m}}{\partial t}\right\|^{2}\right) .
$$

The last two norms on the right hand side are of orders $h^{2 s}$ and $k^{4}$, respectively. Moreover

$$
\begin{aligned}
\left\|f\left(\hat{U}_{m}\right)-\bar{f}\left(u_{m}\right)\right\| & \leq\left\|f\left(\hat{U}_{m}\right)-f\left(u_{m+1 / 2}\right)\right\|+\left\|f\left(u_{m+1 / 2}\right)-\bar{f}\left(u_{m}\right)\right\| \\
& \leq C\left(\left\|\hat{U}_{m}-u_{m+1 / 2}\right\|+k^{2}\right) \\
& \leq C\left(\left\|\hat{\theta}_{m}\right\|+\left\|\hat{\eta}_{m}\right\|+\left\|\hat{u}_{m}-u_{m+1 / 2}\right\|+k^{2}\right) \\
& \leq C\left(\left\|\theta_{m}\right\|+\left\|\theta_{m-1}\right\|+h^{s}+k^{2}\right),
\end{aligned}
$$

where $\hat{\theta}_{m}, \hat{\eta}_{m}$ and $\hat{u}_{m}$ are the extrapolated representations for $\theta_{m}, \eta_{m}$ and $u_{m}$, respectively. On the other hand, the left hand side of (38) is bounded below by

$$
\frac{1}{2} \partial\left\|\theta_{m}\right\|^{2}-\frac{\gamma-1}{2 \gamma} \frac{1}{|\Omega|} \partial\left(\int_{\Omega} \theta_{m} d x\right)^{2} \geq \frac{1}{2 \gamma} \partial\left\|\theta_{m}\right\|^{2} .
$$

Now, in view of (39) and (40), estimate (38) can be written as

$$
\left\|\theta_{m+1}\right\|^{2} \leq(1+C k)\left\|\theta_{m}\right\|^{2}+C k\left\|\theta_{m-1}\right\|^{2}+C k\left(h^{s}+k^{2}\right)^{2},
$$

or

$$
\left\|\theta_{m+1}\right\|^{2}+C k\left\|\theta_{m}\right\|^{2} \leq(1+2 C k)\left(\left\|\theta_{m}\right\|^{2}+C k\left\|\theta_{m-1}\right\|^{2}\right)+C k\left(h^{s}+k^{2}\right)^{2} .
$$

A repeated application of (41), with a small $k$, implies

$$
\left\|\theta_{m}\right\|^{2} \leq C\left(\left\|\theta_{1}\right\|^{2}+k\left\|\theta_{0}\right\|^{2}\right)+C\left(h^{s}+k^{2}\right)^{2} .
$$

If $\theta_{0}$ and $\theta_{1}$ are both calculated with an accuracy $O\left(h^{s}\right)+O\left(k^{2}\right)$, we get the following result

$$
\left\|\theta_{m}\right\| \leq C\left(h^{s}+k^{2}\right),
$$

which proves the following theorem.

Theorem 3. The extrapolated Crank-Nicolson solution $U_{m}$ of (34) satisfies

where $C$ depends on $u$.

$$
\max _{m}\left\|u_{m}-U_{m}\right\| \leq C\left(h^{s}+k^{2}\right),
$$




\section{A GALERKIN METHOD FOR A GASEOUS IGNITION MODEL}

\section{References}

BEBERNES, J. and BRESSAN, A. 1982. Thermal Behaviour for a Confined Reactive gas. J. Diff. Equations, 44: 118-133.

BEBERNES, J. and BRESSAN, A. 1988. Total Blowup Versus Single Point Blowup. J. Diff. Equations, 73(1): 30-44.

BEBERNES, J. and EBERLY, D. 1989. Mathematical Problems from Combustion Theory. Applied Mathematics Series, vol. 83, Springer-Verlag, New York.

BEBERNES, J., BRESSAN, A., KASSOY, D. and RILEY, N. 1989. The Confined Non-Diffusive Thermal Explosion with Spatially Homogeneous Pressure Variation. Comb. Sci. Tech., 63: 45-62.

CANNON, J. and LIN, Y-P. 1990a. A Galerkin Procedure for Diffusion Equations with Integral Boundary Conditions. Int. J. Engng. Sci., 28(7): 579-587.

CANNON, J. and LIN, Y-P. 1990b. A Priori $L^{2}$ error Estimates for Finite Element Methods for Nonlinear Diffusion Equations with Memory. SIAM J. Numer. Anal., 27(3): 595-607.

GILBARG, D. and TRUDINGER, N.S. 1983. Elliptic Partial Differential Equations of Second Order. SpringerVerlag.

KASSOY, D. and POLAND, J. 1983. The Induction Period of a Thermal Explosion in a Gas between Infinite Parallel Plates. Combustion and Flame, 50: 259-274.

THOMÉE, V. 2006. Galerkin Finite Element Methods for Parabolic Problems. Springer Series in Computational Mathematics, Springer-Verlag New York, USA.

Received 21 February 2011

Accepted 15 November 2011 